\title{
The challenges of responsible methods
}

In early September, a scientific committee convened by the United States National Research Council released a report concluding that publication of pathogen genome data should continue. This Committee on Genomics Databases for Bioterrorism Threat Agents argued that the benefit of making these data publicly available outweighs the risk of their being used to devise more potent biological weapons. The creation of this discussion panel is the most recent indication that the life science community is reflecting on the risks of publishing certain types of research. Along with publication of pathogen sequence data, the methods sections of certain papers have repeatedly been singled out at the center of the controversy on the potential dual use of scientific information, making this debate directly relevant to Nature Methods. This journal is committed to fulfilling its goals of communicating important technological advances and doing so responsibly, relying on editorial procedures established to identify and debate potential security issues.

In the wake of the terrorist attacks of September 11,2001 , and the ensuing anthrax-tainted letters sent through the US postal service, the White House, as well as individual scientists, initially proposed measures to withhold from publication details of biotechnological studies, such as sequences and methods, in certain vaguely defined areas of research. Luckily, these extreme propositions triggered a concerted effort on the part of the scientific community to reach a more thoughtful consensus. In February 2003, a group of editors of scientific journals released a joint statement reaffirming that the integrity of the scientific process relies on the publication of manuscripts containing sufficient details to be reproduced. They also made a public commitment to deal responsibly with security issues, recognizing that in some cases the potential harm of publication may outweigh the potential social benefit. They endorsed a model of self-governance, in which the scientists and their journals are responsible for determining the appropriate dissemination of scientific information.

For methods journals such as this one, the concept of stripping a paper of information critical to the reproducibility of the method is a non-starter. There is no point in publishing a method if it cannot be used. But the line between what constitutes a useful tool intended to enhance scientific progress and one that could be used to wreak havoc is extremely difficult, if not impossible, to draw. For us, the problem of duality is even more acute because changing the object of the methodological procedure may be sufficient to change its use. For instance, the same techniques that are used to create aerosols for optimal vaccine delivery can be used to 'weaponize' an infectious agent; even finding an appropriate way to deliver microbial pathogens in aerosol form may be crucial for realistic assessments of vaccination efficacy in animal studies.

Moreover, the beneficial consequences of a method applicable to multiple areas of research will often tip the balance in favor of publication. Reversing this argument by extending the definition of 'sensitive' methodological information to fundamental research tools would be a dangerous obstacle to technological progress. The fact that such fundamental tools as oligonucleotides can be used to reconstitute the genome of small viruses is not a sufficient argument to prevent publication of techniques to facilitate oligonucleotide production. Because of the extreme duality of use, restricting the dissemination of methods must be considered in the light of damage that could be done not only by misusing the technology but also by depriving the scientific community of a tool that could drive progress with enormous public health benefit.

As a Nature journal, in case of potential dual-use information, Nature Methods will adhere to the editors' joint statement of 2003 and will exercise a special review process overseen by an internal committee and involving a network of advisors on security issues. Nevertheless, it remains a complex case-bycase problem, and in the methodological field particularly, there will never be a clear line separating the 'good' methods from the 'bad'. Thus, it is important to continue the dialog between the main stakeholders in this debate: the scientific community, the security community and the general public. Of these groups, scientists are the most aware of the potential deleterious consequences of withholding information, but they are also well armed to evaluate possible misuse of information. It is crucial that they continue to listen to the concerns of those accustomed to dealing with risks to public safety and that they demonstrate to the public a responsible commitment to dealing with these issues. No matter how awkward the dialog may be, it is critical that all parties actively engage in it, for the sake of general security as well as the integrity of the scientific process. 